\title{
The Electrical Brain Activity in Men with Different Alpha-Rhythm Characteristics during Manual Movements Executed by the Subdominant Hand
}

\author{
Olga Korzhyk Olena Morenko Alevtyna Morenko Ihor Kotsan \\ Lesia Ukrainka Eastern European National University, Lutsk, Ukraine
}

\section{Keywords}

Electrical activity · Individual a-frequency - Cerebral cortex · Manual movements $\cdot$ Power $\cdot$ Coherence $\cdot$ Men

\begin{abstract}
Background: The ability to control motor actions and flexibly interact with the environment is considered one of the main components of the human brain executive functions. The spontaneous electroencephalogram (EEG) is among the physiological techniques making it possible to formulate a direct estimation of specific features of the activity of the human brain during manual movements. Purpose: This study is devoted to an investigation of brain processes in men with a high or a low individual a-frequency determined during manual movements executed by the subdominant hand. Methods: A test group consisting of 104 right-handed healthy men from the ages of 19 to 21 was divided into 2 groups in terms of the average magnitude of their individual a-frequency (laF) - groups with high ( $n=53$, laF $\geq 10.04$ $\mathrm{Hz})$ and low $(n=51, \mathrm{laF} \leq 10.04 \mathrm{~Hz})$ values of laF. The power and coherence of the electrical activity of the cerebral cortex as well as the differences between the groups were evaluated by the testees during manual movements executed by
\end{abstract}

the subdominant hand. Results: Manual movements executed by the subdominant hand in response to the sensory signals are generally accompanied by the increased coherence of the EEG frequency components, especially, in the frontal, anterior temporal and central brain regions in men with different a-activity characteristics. Under these conditions, it has been found some electrogenesis power lowering in the cortical areas responsible for the sensory analysis, motor programming, sensory and motor information integration. Such changes have been combined with the local power increase of $\theta-$, a1-oscillations in the frontal leads. Additionally, men with a low laF were characterized by the local growth of a3-activity in the frontal areas of their cortex. Men from both groups also had the generalized increase in the capacity of the high-frequency $\beta 2$ - and $\gamma$-oscillations. Some higher power and coherence of the EEG frequency components have been registered in men with the low laF in comparison with men having some high a-frequency. Conclusion: The functional content of the established differences may generally reflect some relatively lower tone of the cortex activation in men with a low laF and can be specifically compensated by some increased "intensity" and the redundancy of brain processes.

(c) 2018 S. Karger AG, Basel

\section{KARGER}

(c) 2018 S. Karger AG, Basel 


\section{Introduction}

The implementation of manual movements, for example, while we are driving the car, operating gadgets or doing them during social interactions, sports and in everyday life is impossible without the constant renewal and cancellation of motor programs. The ability to control the actions and flexibly interact with the environment is considered one of the main components of the human brain executive functions by a number of authors [1-6]. Researchers [1-6] do not exclude the fact that there are certain indicators of the brain, which are correlated with manual movements.

Many scientists [7-10] have found out the electroencephalogram EEG $\alpha$-rhythm individual variation of the amplitude and frequency characteristics, including the modal frequency of such a rhythm characterized by the highest information to determine the state of the nervous processes and a number of human physiological functions. People, who differ by the $\alpha$-rhythm characteristics, have different behavioral strategies, perception mechanisms and information processing $[7,11]$. According to data obtained by Bazanova and Aftanas [12], any probability of the most optimal process of the coordination of movements linearly correlates with the power of the individual $\alpha$-EEG range and inversely with the tension of the forehead muscles that are not involved in the implementation of voluntary manual movements. Kristeva et al. [13] give evidence that the combination of the synchronization of the neuronal ensembles (the a-EEG amplitude increase) and the economical use of the muscular system (the integrated power reduction of the electromyogram [EMG] of facial muscles) shows an increased capacity of the self-control of movements. Such statements are based on the fact that EEG $\alpha$-modal frequency is believed [14-18] as strictly determined genetic basis, as it reflects the important structural innate characteristics of the thalamic and cortical neurons, in particular, the features of the ionic processes in these cells [19-21].

Defining the critical importance of results obtained by different scientists, it should be emphasized that such a sort of information is clearly insufficient for a comprehensive understanding of the fact how the innate aspect of the brain function as a modal frequency of the EEG $\alpha$-rhythm is connected with the control activities carried out by the distal muscles of the hand during the performance of manual movements. In prior studies [22-24] and in the context of such issues, there were obvious features of brain processes being essential in the control of manual movements performed by the response hand of men with different baseline characteristics of the EEG alpha-rhythm, in particular, the alpha-rhythm modal frequency. According to the results available from experiments, testees with the original high EEG $\alpha$-rhythm modal frequency had higher levels of their selective attention and more local changes of the electrical activity of the cerebral cortex in the regulation of the flow of manual movements. People with a low $\alpha$-frequency are characterized by less specific and differentiated cortex activation processes. It should be emphasized that any creation of the full understanding of features of the human brain processes associated with the manual movements provides the study of such movements being carried out by the fingers of not only the response, but also the subdominant hand. Thus, in real life, people use either their response or subdominant hand during the manual movements. The purpose of this study is to identify the spectral-frequency findings of the cerebral cortex electrical activity of movements performed by men with different characteristics of alpha-activity with the help of their subdominant hand. We believe that the realization of this goal can afford to set more objective indicators of the brain activity being directly associated with the programming of the manual movements.

\section{The Aim of the Study}

It consists of the detection of the spectral-frequency findings of the electrical activity of the cerebral cortex during movements executed by the fingers of the male subdominant hand.

\section{Methods}

\section{Participants}

The participants in our study were 104 male volunteers belonging to the age range 19-21 and each of them had given written consent to be part of the study. Biomedical ethics rules in accordance with the Helsinki Declaration of the World Medical Association on the Ethical Principles of Scientific and Medical Research involving Human Subjects were adhered to during the experiment. All the testees were healthy and had normal hearing with regard to the judgement and advisory conclusions of their medical professionals.

\section{Procedure}

Psychophysiological Examination

As part of the psychophysiological testing, the profile of the manual and auditory asymmetry was determined for each subject. It was determined by the nature of responses obtained from the survey, through the execution of the motor and psychoacoustic tests and by counting the individual ratio of the manual and auditory asymmetries ( $\mathrm{K}_{\text {skew }}$; form 1$)$ [25]. 


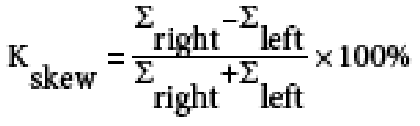

where $\Sigma_{\text {right }}$ is the amount of tasks where a right hand (right ear) is dominating during their execution and $\Sigma_{\text {left }}$ is the amount of tasks under which the left hand (left ear) is dominant.

Further studies involved dextral testees whose coefficients of manual and auditory asymmetries were positive and were above $50 \%$. The total number of men was of 104 people.

All examinations were performed in the morning. The profile of the asymmetry was evaluated 30 min before the EEG recording registration. This made it impossible for it to have any influence on the experiment, particularly, on EEG results.

\section{EEG Testing Procedures}

The testees were in a quiescent state with their eyes closed and in a reclining position with their limbs relaxed and not crossed during the EEG testing. The experiment was carried out in a room that was sound-proof and light-proof. The whole experimental procedure consistently included the following steps for each testee:

Step 1. The EEG recording in the functional balance (background).

Step 2. The EEG recording while performing the finger movements of the left (subdominant) hand.

Each step lasted $40 \mathrm{~s}$. To exclude the edge effects, the EEG recording registration was started $15 \mathrm{~s}$ after the beginning and was stopped $5 \mathrm{~s}$ after its completion.

In order to reduce the stereotypic nature of the test, the sequence of movements was reported to the testees just a few minutes before the test. Finger movements comprised the bending and unbending of the fingers. Each finger's flexion or extension was performed by the testees in response to the sound. The electronic version of the drum battle (the software of Finale 2006) was used for this purpose. Binaural stimuli were produced by 4 speakers placed in different corners of the room at a distance of $1.2 \mathrm{~m}$ from the testee's right or left ear. The stimulus duration was 130 $\mathrm{ms}$; the playback sound volume did not exceed 55-60 dB at outlet from the speakers under the measurements carried out by the sound level meter of the "DE-3301" type (certificate of attestation $\# 025-2009$, valid until December 21, 2014). Additionally, the sound loudness was individually regulated for each testee to achieve the necessary level. The rate of the sound stimuli delivery was $2 \mathrm{~Hz}$.

Registration and Primary Analysis of EEG Data

Active electrodes were placed in accordance with the international system 10/20 in 19 points on the scalp of the head during the electroencephalogram (EEG "Neurocom," and the Certificate of State registration \#6038/2007, valid until April 18, 2014) recording. The performance of the EEG recording was monopolar, with the use of ear electrodes as a reference. The Fourier analysis era was $4 \mathrm{~s}$ with a $50 \%$ overlap. The duration of sample was $40 \mathrm{~s}$. Independent Component Analysis was used for the rejection of EEG anomalies.

Both the power $\left(\mu \mathrm{V}^{2}\right)$ and the coherence of the brain electrical activity in the $\theta-, \alpha-, \beta$-, and $\gamma$-frequency intervals were also evaluated. Taking into consideration the functional heterogeneity of different sub-bands of the EEG $\alpha$ - and $\beta$-rhythms, the changes in the power and coherence of each of them were considered, and coefficients of coherence above 0.5 were analyzed as well.

The maximum frequency peak of the $a$-rhythm was determined for each testee in each EEG lead at a functional balance [26]. Its value was averaged for all leads and the obtained values were considered the testee's individual $\alpha$-frequency (the individual alpha-frequency of EEG, individual $\alpha$-frequency [I $\alpha \mathrm{F}]$, and $\mathrm{Hz}$ ). The I $\mathrm{F}$ median was also determined and calculated for the group of men. It was $10.04 \mathrm{~Hz}$. Thus, subgroups of testees were formed according to the value of the median:

- Subgroup with a high $\operatorname{IaF}(n=53, \mathrm{I} \alpha \mathrm{F} \geq 10.04 \mathrm{~Hz})$;

- Subgroup with a low IaF $(n=51, \mathrm{I} \alpha \mathrm{F}<10.04 \mathrm{~Hz})$.

The EEG frequency interval limits were determined individually, relying on the value of the testee's IaF. The following algorithm [26] was used; the truth was that the upper limit of a3subband was set to the right side of the $\mathrm{I} \alpha \mathrm{F}$ in increments of $2 \mathrm{~Hz}$. It corresponded to the lower limit of the $\beta 1$-band. The upper limit of the $\beta 1$-sub-band was defined according to the standard concepts as $25 \mathrm{~Hz}$. The lower limit of the $\mathrm{a} 2$ - band was determined in steps of $2 \mathrm{~Hz}$ to the left of the peak, and the a1band in $4-\mathrm{Hz}$ steps, as well as $\theta$-frequencies - in $6 \mathrm{~Hz}$. Limits of $\beta 2$ - and $\gamma$-bands were recognized as standard, properly, 26-35 and $36-45 \mathrm{~Hz}$.

The resulting individual values of the power and coherence of EEG oscillations within the selected groups of men were averaged for each lead.

\section{Statistical Analyses}

A statistical data analysis was performed by using the package "STATISTICA 6.0" (Stat-Soft, 2001). Any normalcy of the data distribution in testees' subgroups was evaluated using the Shapiro-Wilks test (indicator SW). Based on test results, it was found that all of our studied samples had a normal data distribution. To estimate the significance of differences existing in testees' subgroups, the Student $t$ test (index $t$ ) was used between steps of testing both for independent equal samples and for dependent samples. Significant differences between testees' subgroups and among steps of testing were statistically considered at $p \leq 0.05$ and $p \leq 0.01$.

\section{Results}

\section{The Individual Modal Frequency Evaluation of the $\alpha$-EEG Activity and Individual Limits of the Frequency Content of the EEG Sub-Range in Male Testees Findings}

The average value of the modal frequency of any a-activity in samples of male testees was $10.04 \pm 0.03 \mathrm{~Hz}$. Considering the leveled nature of the individual a-frequency value histogram (Fig. 1) in the male testees, it was made the conditional distribution of samples under the average mean of the modal frequency of $\alpha$-activity. Two groups were formed - one group having a high value of $\mathrm{IaF}(n=53, \mathrm{I} \alpha \mathrm{F} \geq 10.04 \mathrm{~Hz})$ and another group with a low value of $\mathrm{I} \alpha \mathrm{F}(n=51, \mathrm{I} \alpha \mathrm{F}<10.04 \mathrm{~Hz})$. 
Fig. 1. Histogram of values of $a$-frequency mode in male testees. Vertical columns individual values of the EEG a-frequency mode in samples involving male testees.

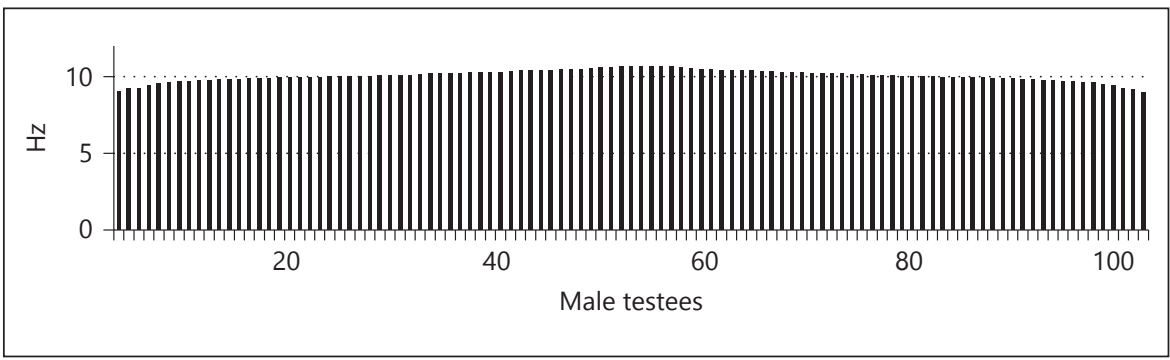

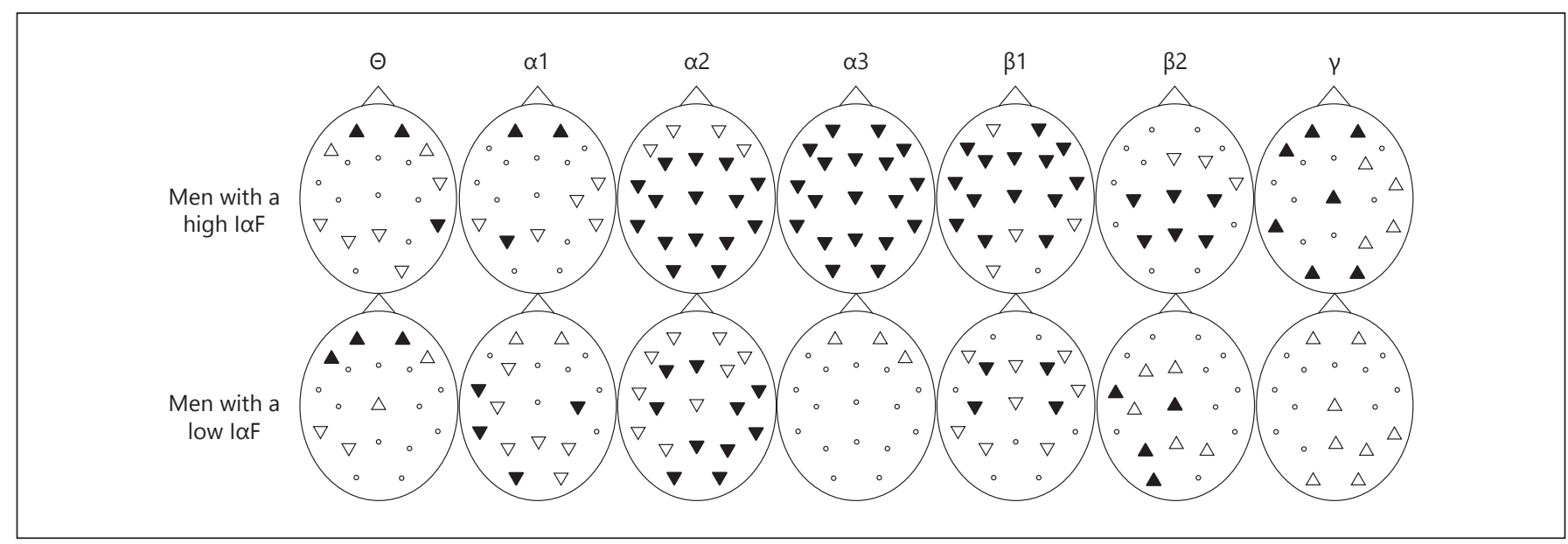

Fig. 2. Topo maps of changes of EEG power fluctuations through the execution of manual movements by male groups. $\triangle \Delta \nabla \nabla$ Increase (triangle up)/decrease (triangle down) of EEG power fluc- tuations through the execution of manual movements compared to power in a quiescent state, $p \leq 0.05$ (white triangle), $p \leq 0.01$ (black triangle).
Features of the Cortical Activity While Performing the Fingers Movements in Men Having a Different I $\alpha F$

Men with a high I $\alpha \mathrm{F}$ are characterized by the increasing local capacity of the EEG $\theta$ - $\alpha 1$-oscillations in the frontal areas. Apparently, according to Klimesch et al. [26], Lurija [27], Buzsáki [28] and Avery et al. [29], such changes may be the result of evincing some closer voluntary attention, actualizing operative memory traces and keeping the focus on information concerning the sensory stimuli and movements being performed. Corresponding changes were more generalized in $\gamma$-band activity (with $p \leq 0.05$ and $p \leq 0.01$; Fig. 2).

The diffuse increase of the capacity of $\gamma$-oscillations in the cortex can be considered a criterion easing out the interaction of widely distributed neural networks involved in information processing and in some sense-motor integration [30]. Instead of it, some power decrease of the EEG $\theta$-, $\alpha$ - and $\beta$-waves, especially at the rear cortical areas (with $p \leq 0.05$ and $p \leq 0.01$ ) has been observed. This regularity had some higher prevalence and significance in the cortex in the range of $\alpha 2-, \alpha 3$ - and $\beta 1$-frequency components $(r \leq 0.01)$. According to the literature references $[26-28,31]$, the reducing power of the electrical activity can indicate the increased activity of the cortical areas. Under the terms of researches, the activation processes found in cortical structures can display their participation in the processes of the sensory analysis, motor programming and integration of some sensory and motor information.

Men with a low IaF had the power increase of $\theta-$, $\alpha 1-$ and $\alpha 3$-activity locally in the frontal areas (with $p \leq 0.05$ and $p \leq 0.01$ ), and $\beta 2$ - and $\gamma$-waves are mostly generalized in the cortex ( $r \leq 0.05$; Fig. 2$)$. The presence of some power increase of $\alpha 3$-waves in the frontal area $(r \leq 0.05)$ in men with a low I $\alpha \mathrm{F}$ can display the additional braking mechanisms of the sensory input $[29,32]$ and can be the EEG-correlator of the matching process of the afferent information flows as to the new parameters of the muscle activity with the downgoing effects of the frontal cortex on the previous motor program. Such descending inhibi- 


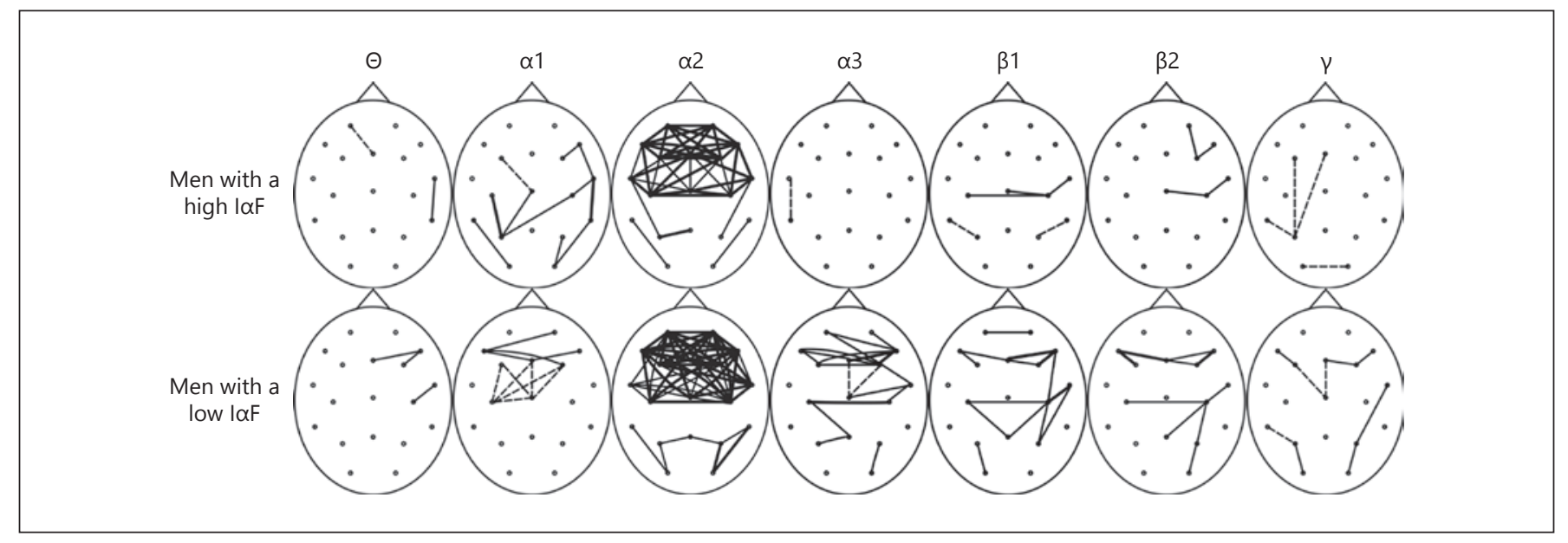

Fig. 3. Topo maps of changes of EEG coherence oscillations through the execution of manual movements by male groups. ,$--1(---,---)$ Increase (solid line)/decrease (dotted line)

tory influences generally prevent the motor programming [1] in the course of manual movements performed by the subdominant hand and, according to Kostandov [32], they show some less flexibility of commands.

The reduction of the capacity in the cortex at frequency of the EEG $\theta-, \alpha 1-, \alpha 2-, \beta 1$-oscillations (with $p \leq 0.05$ and $p \leq 0.01$ ) was specified both in men with a high $\mathrm{I} \alpha \mathrm{F}$ and in men with a low a-frequency. However, these changes were relatively more local and less significant.

Some increased coherence of the EEG a1-, a2- and $\beta$-oscillations was recorded in men with a high individual $\alpha$-frequency in the frontal, temporal and central parts of their cortex $(p \leq 0.05)$. Such a regularity has the greatest significance in $\alpha 2$-subdiapason (with $p \leq 0.05$ and $p \leq$ 0.01; Fig. 3).

According to Lurija [27], it may be associated with the functional association of the cortical areas involved in the control of movements performed by the distal muscles with the increased voluntary control. Under the results of Zhavoronkova [25], this feature, in terms of movements performed by the subdominant hand, can be compensatory in nature and facilitate the spread of some excitement among different cortex areas. Simultaneously, a decrease of the coherence of the $\theta_{-}, \alpha 1-, \alpha 3-, \beta 1-$ and $\gamma$-activity having quite a local character in the cortex of the male testees was found out. Such changes were observed in the anterior cortical areas $(p \leq 0.05)$ in the range of the low $\theta$ - and $\alpha 1$-frequency, and the reduction of the coherence - mainly in the posterior cortical structures $(p \leq 0.05)$ in the high frequency range of the EEG ( $\beta$ - and $\gamma$-activity). of EEG coherence oscillations through the execution of manual movements compared with the coherence in a quiescent state, $p \leq$ 0.05 (a thin line), $p \leq 0.01$ (a thick line).

Some increased coherence coefficients in the cortex has relatively greater prevalence and importance in the cortex especially at a frequency of $\alpha 2-, \alpha 3-, \beta$ - and $\gamma$-activity (with $p \leq 0.05$ and $p \leq 0.01$ ) in men with a low individual $\alpha$-frequency than in men with a high IaF (Fig. 3). At the same time, the reduction in coherence was primarily found out in the anterior cortical areas in the strip of the EEG $\alpha 1-, \alpha 3$ - and $\gamma$-oscillations $(p \leq 0.05)$.

\section{Intergroup Differences}

In terms of manual movements performed by the left subdominant hand, men with a low I $\alpha$ F have some higher power of the EEG frequency components in comparison with men having a high $\alpha$-frequency (Fig. 4). Such a generalized regularity was observed in the cortex (with $p \leq 0.05$ and $p \leq 0.01$ ). According to Iakovenko et al. [33] such a regularity may indicate some slightly higher tone of the cortex activation associated with the control of the executive and information processes in people with a high IaF. Men with a low I $\alpha \mathrm{F}$ are characterized by the higher coefficients of the coherence in the frequency range of the front and rear cortical areas (with $p \leq 0.05$ and $p \leq 0.01$ ). Conditions easing out the compensatory spread of some excitement among individual "nodes of the structural and functional perception systems" may be created against the background of a slightly lower tone of the functional cortex in this group of testees [33]. The functional content of this spatial synchronization type in the cortex is associated with higher levels of some "tension" and the redundancy of brain processes [34]. 


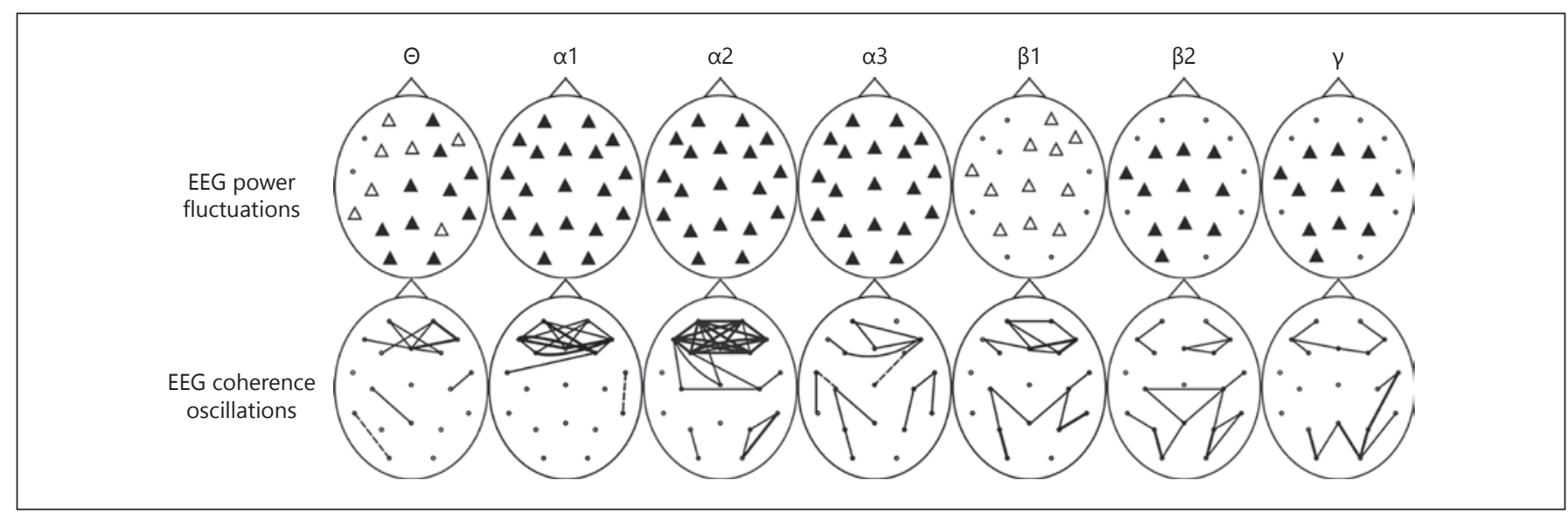

Fig. 4. Intergroup differences in EEG power fluctuations and in EEG coherence oscillations through the execution of manual movements. $\triangle \boldsymbol{\Delta} \boldsymbol{\nabla} \nabla$ Higher (lower) power in men with a low I $\alpha \mathrm{F}$ in comparison with men with a high $\mathrm{I} \alpha \mathrm{F}, p \leq 0.05$ (white triangle), $p \leq 0.001$ (black triangle). $\_,-/(---,---)$Higher (lower) coherence in men with a low $\mathrm{I} \alpha \mathrm{F}$ in comparison with men having a high I $\alpha$ F, $p \leq 0.05$ (a thin line), $p \leq 0.001$ (a thick line).

\section{Conclusions}

Thus, any finger closing and unclosing performed by the subdominant hand of men with different $\alpha$-activity characteristics in response to the sensory signals were generally accompanied by the increased coherence of the EEG frequency components, particularly, in the frontal, anterior temporal and the central areas and could be compensatory in nature and facilitate the spread of some excitation among different areas of the cortex. Under these conditions, the reduced electrogenesis power in the cortical areas responsible for the sensory analysis, motor programming and integration of sensory and motor information was observed. Such changes were combined with the local power increase of $\theta-$, al-oscillations in the frontal leads, primarily due to the strengthening of some voluntary attention and memory processes. Additionally, men with a low IaF had a local growth of $\alpha 3$-activity in the frontal areas of their cortex, which is obviously a sign of the additional braking mechanisms of the sensory input during the motor programming. The evidence of the sense-motor integration deepening in the result of the interaction of the distant neural networks was seen in men from both groups characterizing by some increase generalized in the capacity of the high $\beta 2$ - and $\gamma$-oscillations. Men with a low IaF had some higher power and frequency of EEG coherence components compared to men with a high $a$-frequency. The functional content of such differences may relatively reflect the lower tone of the cortex activa- tion in men with a low IaF and it can be compensated by the increased "intensity" and redundancy of brain processes.

\section{Ethical Statement}

Biomedical ethics rules in accordance with the Helsinki Declaration of the World Medical Association on the Ethical Principles of Scientific and Medical Research involving Human Subjects were adhered to during the experiment.

\section{Disclosure Statement}

Authors do not have any conflicts of interest to disclose.

\section{Funding Source}

Work has been performed under the research project using the state budget funds of Ukraine, reg. No. 0111 U002143.

\begin{tabular}{|c|c|}
\hline References & $\begin{array}{l}\text { Ioffe ME: [Neural basis of learning new move- } \\
\text { ments: evolution of classical concepts]. Zh } \\
\text { Vyssh Nerv Deiat Im I P Pavlova 2003;53:5-21. } \\
2 \text { Ruge D, Muggleton N, Hoad D, et al: An un- } \\
\text { avoidable modulation? Sensory attention and } \\
\text { human primary motor cortex excitability. Eur } \\
\text { J Neurosci 2014;40:2850-2858. } \\
\text { 3 Averbeck BB, Chafee MV, et al: Parallel process- } \\
\text { ing of serial movements in prefrontal cortex. } \\
\text { Proc Natl Acad Sci U S A 2002;99:13172-13177. }\end{array}$ \\
\hline
\end{tabular}


4 Cisek P: Neural representations of motor plans, desired trajectories, and controlled object. Cognit Proc 2005;6:15-24.

5 Hikosaka O, Nakamura K, et al: Central mechanisms of motor skill learning. Curr Opin Neurobiol 2002;12:217-222.

-6 Müller GR, Neuper C, et al: Event-related beta EEG changes during wrist movements induced by functional electrical stimulation of forearm muscles in man. Neurosci Lett 2003; 340:143-147.

7 Bazanova OM: Age related alpha activity change differs for males and females and for low and high alpha frequency EEG patterns. Revista Española de Neuropsicología 2008; 10:82-83.

$>8$ Kaplan AIa, Borisov SV: Dynamic properties of segmental characteristics of EEG alpha activity in rest conditions and during cognitive tasks. Zh Vyssh Nerv Deiat Im I P Pavlova 2003;53:22-32.

$>9$ Razumnikova OM, Tarasova IV, Vol'f NV: [Characteristics of cortical activity in persons with high and low verbal creativity: analysis of alpha1,2 rhythms]. Zh Vyssh Nerv Deiat Im I P Pavlova 2009;59:581-586.

10 Umriukhin EA, Korobeĭnikova II, Karatygin NA: [Success of the task performance by students with different spectral alpha-rhythm characteristics in the baseline electroencephalogram]. Fiziol Cheloveka 2009;35:33-39.

-11 Hummel F, Saur R, Lasogga S, et al: To act or not to act. Neural correlates of executive control of learned motor behavior. NeuroImage 2004;23:1391-1401.

$>12$ Bazanova OM, Aftanas LI: [Individual alpha activity of electroencephalogram and nonverbal creativity]. Ross Fiziol Zh Im I M Sechenova 2007;93:14-26.

13 Kristeva R, Chakarov V, Losch F, et al: Electroencephalographic spectral power in writer's cramp patients: evidence for motor cortex malfunctioning during the cramp. Neuroimage $2005 ; 27: 706-714$
14 Anderson MP, Mochizuki T, Xie J, et al: Thalamic Cav3.1 T-type $\mathrm{Ca} 2+$ channel plays a crucial role in stabilizing sleep. Proc Natl Acad Sci U S A 2005; 102:17431748.

15 Page AJ, O’Donnell TA, Blackshaw LA: Inhibition of mechanosensitivity in visceral primary afferents by $\mathrm{GABAB}$ receptors involves calcium and potassium channels. Neuroscience 2006;137:627-636.

16 Spergel DJ: Calcium and small-conductance calcium-activated potassium channels in gonadotropin-releasing hormone neurons before, during, and after puberty. Endocrinology 2007; 148:2383-2390.

17 Anokhin AP, Muller V, Lindenberger U, et al: Genetic influences on dynamic complexity of brain oscillations. Neurosci Lett 2006;397:9398.

18 Smit CM, Wright MJ, Hansell NK, et al: Genetic variation of individual alpha frequency (IAF) and alpha power in a large adolescent twin sample. Int J Psychophysiol 2006;61: 235-243.

19 Bellone C, Nicoll RA: Rapid bidirectional switching of synaptic NMDA receptors. Neuron 2007;55:779-788.

20 Ng SC, Raveendran P: EEG peak alpha frequency as an indicator for physical fatigue. Medicon 2007;16:517-520.

$>21$ Niswender CM, Jones CK, Conn PJ: New therapeutic frontiers for metabotropic glutamate receptors. Curr Top Med Chem 2005;5: 847-857.

22 Morenko A, Tsos A, Kocan I: Features of the cortical activity of men having a high or low alpha-frequency background of the EEG while performing alternate finger movements. Health Probl Civilization 2014;8:2431.

23 Morenko A, Tsos A, Kocan I: Electrical activity of the cerebral cortex in men having high or low output alpha-frequencies while performing usual manual movements in re- sponse to sensory signals. Human Health 2013;7:3-9.

24 Morenko AG, Pavlovych OS, Kotsan IIa: [Features of cortical activation processes in men during auditorimotor activity of different complexity]. Fiziol Zh 2013;59:41-49.

25 Zhavoronkova L: Right-Handed People, The Left-Hander. Hemispheric Asymmetry of the Human Brain Biopotentials. Krasnodar, Science, 2009.

26 Klimesch W, Sauseng P, Hanslmayr S: EEG alpha oscillations: the inhibition-timing hypothesis. Brain Res Rev 2007;53:63-88.

27 Lurija AR: Higher Cortical Functions of Man and their Disturbances in Local Brain Lesions. Moskva, Akademicheskiy Proekt, 2000.

28 Buzsáki G: Rhythms of the Brain. New York, Oxford University Press, 2006.

29 Avery MC, Dutt N, Krichmar JL: Mechanisms underlying the basal forebrain enhancement of top-down and bottom-up attention. Eur J Neurosci 2014;39:852-865.

30 Pulvermuller F, Birbaumer N, Lutzenberger W, et al: High-frequency brain activity: its possible role in attention, perception and language processing. Prog Neurobiol 1997;52: 427-445.

31 Tebenova K: Study of bioelectric activity of the brain in operators of telephone stations. Sovremennyje problemy nauki I obrazovanija 2009;4:138-141.

32 Kostandov EA: [Flexibility of cognitive activity depends on its context]. Fiziol Cheloveka 2010;36:19-28.

33 Iakovenko IA, Cheremushkin EA, et al: [Wavelet transformation of event-related cortical activity during formation of visual set to emotional face image]. Zh Vyssh Nerv Deiat Im I P Pavlova 2010;60:409-418.

34 Kiroı̆ VN, Belova EI: [The Mechanisms of the formation and the role of the oscillatory activity of the neuronal populations in brain systemic activities]. Zh Vyssh Nerv Deiat Im I P Pavlova 2000;50:179-191. 